\title{
Prevalence of Postprostatectomy Incontinence Requiring Anti- incontinence Surgery After Radical Prostatectomy for Prostate Cancer: A Retrospective Population-Based Analysis
}

\author{
Jae Heon Kim ${ }^{1,2}$, In Gab Jeong ${ }^{1,3}$, Yash S. Khandwala ${ }^{1}$, Tina Hernandez-Boussard ${ }^{4}$, James D. Brooks ${ }^{1}$, Benjamin I. Chung ${ }^{1}$ \\ ${ }^{1}$ Department of Urology, Stanford University Medical Center, Stanford, CA, USA \\ ${ }^{2}$ Department of Urology, Soonchunhyang University Hospital, Soonchuhyang University Medical College, Seoul, Korea \\ ${ }^{3}$ Department of Urology, Asan Medical Center, University of Ulsan College of Medicine, Seoul, Korea \\ ${ }^{4}$ Department of Biomedical Informatics, Stanford University Medical Center, Stanford, CA, USA
}

\begin{abstract}
Purpose: This study aimed to examine the prevalence of surgery for postprostatectomy incontinence (PI) following minimally invasive surgery compared to conventional open surgery for prostate cancer.

Methods: This retrospective cohort study used the Florida State Ambulatory Surgery and State Inpatient Databases, 2008 to 2010, radical prostatectomy (RP) patients were identified using International Classification of Diseases (ICD)-9/10 procedure codes and among this cohort, PI was identified also using ICD-9/10 codes. Surgical approaches included minimally invasive (robotic or laparoscopic) versus open (retropubic or perineal) RP. The primary outcome was the overall prevalence of surgery for PI. The secondary outcome was the association of PI requiring anti-incontinence surgery with the surgical approach for RP.

Results: Among the 13,535 patients initially included in the study (mean age, 63.3 years), 6,932 (51.2\%) underwent open RP and 6,603 (49.8\%) underwent minimally invasive RP. The overall prevalence of surgical procedures for PI during the observation period among the all patients who had received RP was 3.3\%. The rate of PI surgery for patients receiving minimally invasive surgery was higher than that for patients receiving open surgery (4.8\% vs. 3.0\%; risk difference, $1.8 \%$; $95 \%$ confidence interval, $0.3 \%-3.4 \%$ ). The adjusted prevalence of PI surgery for patients who had undergone laparoscopic RP was higher than that for those with retropubic RP ( $8.6 \%$ vs. $3.7 \%)$.

Conclusions: Among patients undergoing RP for prostate cancer, the prevalence of PI surgery is not negligible. Patients undergoing minimally invasive RP had higher adjusted rates for PI surgery compared to open approaches, which was attributed to high rate of PI surgery following laparoscopic approach and low rate of PI surgery following perineal approach. More studies are needed to establish strategies to reduce the rate of PI surgery after RP.
\end{abstract}

Keywords: Prostatic neoplasm; Prostatectomy; Urinary incontinence; Robotic surgical procedures; Laparoscopy

- Fund/Grant Support: This work has been supported by Soonchunhyang University Research Fund.

- Conflict of Interest: No potential conflict of interest relevant to this article was reported.

Corresponding author: In Gab Jeong (iD https://orcid.org/0000-0000-0000-0000 Department of Urology, Asan Medical center, University of Ulsan College of Medicine, 88 Olympi-ro 43-gil, Songpa-gu, Seoul 05505, Korea Email: igjeong@amc.seoul.kr

Submitted: April 14, 2020 / Accepted after revision: September 23, 2020
This is an Open Access article distributed under the terms of the Creative Commons Attribution Non-Commercial License (https://creativecommons.org/licenses/by-nc/4.0/) which permits unrestricted non-commercial use, distribution, and reproduction in any medium, provided the original work is properly cited. 


\section{INTRODUCTION}

Radical prostatectomy (RP) has been a standard treatment for localized prostate cancer (PCa) [1]. Although the oncological benefit of RP has been demonstrated in prospective randomized trials, particularly for men with intermediate and high-risk disease, patient quality of life (QoL) can be affected by postoperative complications that may include erectile dysfunction (ED) and postprostatectomy incontinence (PI) [2].

PI has been demonstrated to significantly reduce patient QoL [3], far outranking ED with respect to patient dissatisfaction following RP [4]. Although PI is of concern to surgeons and patients alike, investigations have reported varied prevalence of PI following RP. This is likely attributable to the accepted belief that PI will recover over time. Indeed, previous investigations have reported that $68 \%$ of patients regain complete continence by 2 months postoperation, with this number increasing to $\sim 90 \%$ at 12 - and 18-month postoperation $[5,6]$. However, several studies have indicated various rates of persistent PI ranging from $4 \%-30 \%$, according to the definition of PI used [7]. Some studies have shown the prevalence of PI and their predictors by surgical modality, yet most of these studies were limited by small sample sizes, exclusion of young populations, lack of a detailed surgical approach, and short follow-up duration [5-7]. In view of the long-life expectancy associated with surgically treated PCa, maximizing patient QoL should be considered to be concurrent with PCa-specific survival $[6,8]$. Therefore, the true prevalence of long-standing, severely bothersome PI must be understood into order to select optimal treatment strategies for PCa.

The objective of this study is to examine the prevalence of PI requiring anti-incontinence surgery in patients receiving minimally invasive surgery and to compare this prevalence with conventional open surgery for PCa using large cohort data from the Healthcare Cost and Utilization Project National Inpatient Sample database.

\section{MATERIALS AND METHODS}

\section{Data Source}

A retrospective cohort study was performed using discharge data from Florida State Ambulatory Surgery and Services Databases and State Inpatient Databases, Healthcare Cost and Utilization Project, Agency for Healthcare Research and Quality (www.hcup-us.ahrq.gov/databases.jsp) to examine the preva- lence of PI in patients receiving RP. These are all-capture state databases that contain patient characteristics, primary and secondary diagnoses, and procedures, and these databases afford the ability to link patients over time.

\section{Patients}

Patients receiving RP between January 2008 and December 2010 were identified using the International Classification of Diseases, Ninth Revision, Clinical Modification (ICD-9 CM) procedural code. Patients with metastatic PCa were excluded from the study (ICD-9 CM codes 196, 197, 198, and 199). Due to missing variable information, a further 1,426 patients were excluded. All patients were followed up for at least 2 years after $\mathrm{RP}$ in order to track the prevalence of PI.

\section{Main Exposures}

Patients receiving open (60.5: retropubic or 60.62: perineal) or minimally invasive RP were identified using the ICD-9 procedural code. Using the ICD-9 procedural code or Current Procedural Terminology (CPT) code, minimally invasive surgeries were further differentiated to laparoscopic (ICD-9 CM: 54.21 or CPT: 55866) or robotic-assisted surgery (ICD-9 CM: 17.4x). The presence of PI was defined as the rate of PI surgery in this study and was identified by using affiliated ICD-9 CM procedure codes/CPT codes ensuring the anti-incontinence surgeries. Anti-incontinence therapies included 3 procedures: (1) sling operation; (2) artificial urinary sphincter (AUS) or inflatable device, and (3) bulking agent injection. Sling operation was identified using ICD-9 CM procedure codes (59.3, 59.4, 59.5, 59.6, and 59.7) and CPT codes (53440, 53443, 51840, and 51841). AUS or inflatable device was referenced using ICD-9 CM procedure codes (58.93) and CPT codes (53444, 53445, and 53449). Injection of the bulking agent was referenced using ICD-9 CM procedure codes of 59.72 and CPT code (51715).

\section{Demographic and Clinical Characteristics}

Hospital level data collected included location (urban or rural) and teaching status (teaching or nonteaching). Patient-related data included age, race (white, black, Hispanic, or other), household income (poorest, poor, wealth, wealthiest), and insurance status (private, Medicare, Medicaid, or other). The comorbidity condition of each patient was described using the Charlson Comorbidity Index. Household income was defined by medical household income by quantile distribution using zip codes. The annual hospital volume was estimated according to the number 
of procedures at a given patient's hospital, and was defined as more than the $75 \%$ percentile (highest quartile).

\section{Outcomes}

The primary outcome was the overall prevalence of PI surgery. The presence of PI was defined as the rate of PI surgery in this study and was identified by using affiliated ICD- 9 CM procedure codes/CPT codes ensuring the anti-incontinence surgeries. The secondary outcome was the association between prevalence of PI surgery with the surgical approach for RP (open vs. minimally invasive or retropubic vs. perineal vs. laparoscopic vs robot-assisted RP). The prevalence of PI surgery was defined as having either ICD-9 CM procedure code or CPT code along with or without any relevant ICD-9 CM diagnostic codes.

\section{Statistical Analysis}

Categorical variables were presented as numbers and percentages and were compared using chi-square tests. A weighted logistic regression model with inverse probability of treatment weighting (IPTW) was developed in order to adjust for potential confounders described in Table 1 at the baseline patient characteristics level. The weights used for patients undergoing open RP (retropubic or perineal) were the inverse of ' 1 -propensity score, and weights used for patients receiving minimally invasive RP (laparoscopic RP or robotic-assisted RP) were the inverse of just the propensity score, which were estimated by multiple logistic regression analysis without considering the outcomes. Log-binomial regression models were utilized in order to estimate risk ratios (RRs) for each procedure on PI. All models were adjusted for the clustering of patients within hospitals in order to adjust for interhospital variability. A sensitivity analysis was also conducted to determine whether the rate of PI surgery related to each surgical approach (open and minimally invasive) was influenced by the detailed surgical modality within each surgical approach (retropubic, perineal, laparoscopic, and robotic-assisted RP). During sensitivity analysis, a new IPTW was conducted in order to analyze the RR for each procedure, after adjustment for confounders. These analyses were not prespecified but rather conducted post hoc and should thus be interpreted as exploratory. Statistical analysis was performed using 2-sided tests with a significance level of $<0.05$ and Stata 14 (StataCorp LLC, College Station, TX, USA).

\section{RESULTS}

A cohort of 13,535 patients undergoing elective minimally invasive (robotic-assisted RP: $n=6,100$ and laparoscopic RP: $n=$ 503) or open RP (retropubic: $n=5,173$ and perineal: $n=1,759$ ) has been included for final analysis. The demographic characteristics of this cohort are summarized in Table 1. Before the propensity weighting process, the open and minimally invasive RP cohorts differed in several variables, particularly age, insurance status, and annual hospital volume. Following propensity score weighting, similar covariate distributions were achieved between open and minimally invasive RP in the weighted populations (the standardized difference score was $<0.2$ ).

The overall prevalence of PI surgery during the observation period among those patients who had received RP between 2008 and 2010 was 3.3\%. The crude rate of detailed anti-incontinence procedure was described in Table 2. The most common procedure for first treatment of PI was the sling procedure (1.8\%), followed by injection of a bulking agent $(0.8 \%)$, and AUS in $0.6 \%$ of cases.

The unadjusted prevalence of PI surgery was similar between the open and minimally invasive groups (Table 3). However, the IPTW-adjusted prevalence of PI surgery for patients receiving minimally invasive surgery was higher than for those who received open surgery ( $4.8 \%$ vs. $3.0 \%$; RR, 1.60 ; $95 \%$ confidence interval [CI], 1.16-2.20).

For each of the 4 surgical modalities, the unadjusted prevalence of PI surgery is presented in Table 4. The IPTW-adjusted prevalence of PI surgery for patients receiving perineal RP was lower than that for those with retropubic RP $(0.6 \%$ vs. $4.3 \%$; RR, 0.13; 95\% CI, 0.07-0.23). However, the IPTW-adjusted prevalence of PI surgery for patients receiving laparoscopic RP was higher than for those with retropubic RP ( $8.6 \%$ vs. 3.7\%; RR, 2.36; 95\% CI, 1.20-4.64). The unadjusted and IPTW-adjusted prevalence of PI surgery was similar between retropubic and robotic-assisted RP.

\section{DISCUSSION}

To the best of our knowledge, this study is the first to define the prevalence of PI surgery following RP in a large cohort analysis, while simultaneously investigating for differences in the prevalence of PI surgery according to the initial surgical modality employed, including retropubic, perineal, laparoscopic, and robotic-assisted RP. The main outcome of our study was to pres- 
Table 1. Baseline characteristics of patients receiving open and minimally invasive radical prostatectomy

\begin{tabular}{|c|c|c|c|c|c|c|c|c|}
\hline \multirow[b]{2}{*}{ Characteristic } & \multicolumn{4}{|c|}{ Before propensity weighting } & \multicolumn{4}{|c|}{ After propensity weighting } \\
\hline & $\begin{array}{c}\text { Open } \\
(n=6,932)\end{array}$ & $\begin{array}{c}\text { Minimally } \\
\text { invasive } \\
(n=6,603)\end{array}$ & $\begin{array}{l}\text { Standardized } \\
\text { difference }\end{array}$ & P-value & $\begin{array}{c}\text { Open } \\
(n=6,932)\end{array}$ & $\begin{array}{c}\text { Minimally } \\
\text { invasive } \\
(\mathrm{n}=6,603)\end{array}$ & $\begin{array}{l}\text { Standardized } \\
\text { difference }\end{array}$ & P-value \\
\hline Age (yr) & & & & $<0.001$ & & & & 0.816 \\
\hline$<55$ & $922(13.3)$ & $1,210(18.3)$ & 0.138 & & $1,062(15.3)$ & $1,023(15.5)$ & 0.005 & \\
\hline $55-64$ & $2,473(35.7)$ & $2,834(42.9)$ & 0.149 & & $2,708(39.1)$ & $2,558(38.7)$ & -0.007 & \\
\hline $65-74$ & $2,776(40.1)$ & $2,427(36.8)$ & -0.068 & & $2,692(38.8)$ & $2,540(38.5)$ & -0.008 & \\
\hline$>74$ & $761(10.9)$ & $132(2.0)$ & -0.371 & & $470(6.8)$ & $482(7.3)$ & 0.020 & \\
\hline Race & & & & $<0.001$ & & & & 0.926 \\
\hline White & $4,625(66.7)$ & $4,577(69.3)$ & 0.056 & & $4,669(67.3)$ & $4,477(67.8)$ & 0.010 & \\
\hline Black & $1,087(15.7)$ & $787(11.9)$ & -0.109 & & $1,002(14.5)$ & $928(14.1)$ & -0.012 & \\
\hline Hispanic & $900(13.0)$ & $819(12.4)$ & -0.017 & & $908(13.1)$ & $854(12.9)$ & -0.005 & \\
\hline Others & $320(4.6)$ & $420(6.4)$ & 0.077 & & $353(5.1)$ & $344(5.2)$ & 0.005 & \\
\hline CCI score & & & & 0.187 & & & & 0.872 \\
\hline 0 & $3,358(48.4)$ & $3,266(49.5)$ & 0.020 & & $3,377(48.7)$ & $3,183(48.2)$ & -0.010 & \\
\hline 1 & $2,191(31.6)$ & $1,991(30.1)$ & -0.031 & & $2,153(31.1)$ & $2,059(31.2)$ & 0.003 & \\
\hline$\geq 2$ & $1,383(20.0)$ & $1,346(20.4)$ & 0.011 & & $1,402(20.2)$ & $1,361(20.6)$ & 0.009 & \\
\hline Insurance status & & & & $<0.001$ & & & & 0.900 \\
\hline Medicare & $3,282(47.4)$ & $2,336(35.4)$ & -0.245 & & $2,905(41.9)$ & $2,775(42.0)$ & 0.002 & \\
\hline Medicaid & $149(2.1)$ & $87(1.3)$ & -0.064 & & $136(1.9)$ & $116(1.8)$ & -0.015 & \\
\hline Private & $3,163(45.6)$ & $3,880(58.8)$ & 0.265 & & $3,552(51.3)$ & $3,379(51.2)$ & -0.001 & \\
\hline Others & $338(4.9)$ & $300(4.5)$ & -0.016 & & $339(4.9)$ & $333(5.0)$ & 0.007 & \\
\hline Household income & & & & $<0.001$ & & & & 0.994 \\
\hline Poorest & $1,737(25.1)$ & $1,296(19.6)$ & -0.131 & & $1,560(22.5)$ & $1,490(22.6)$ & 0.001 & \\
\hline Poor & $2,189(31.6)$ & $1,992(30.2)$ & -0.031 & & $2,125(30.7)$ & $2,038(30.9)$ & 0.005 & \\
\hline Wealth & $1,957(28.2)$ & $2,053(31.1)$ & 0.063 & & $2,065(29.8)$ & $1,961(29.7)$ & -0.002 & \\
\hline Wealthiest & $1,049(15.1)$ & $1,262(19.1)$ & 0.106 & & $1,182(17.0)$ & $1,114(16.8)$ & -0.005 & \\
\hline Hospital location & & & & 0.212 & & & & 0.935 \\
\hline Urban & $6,467(93.3)$ & $6,124(92.7)$ & -0.021 & & $6,479(93.5)$ & $6,169(93.4)$ & -0.002 & \\
\hline Rural & $465(6.7)$ & $479(7.3)$ & & & $453(6.5)$ & $434(6.6)$ & & \\
\hline Annual hospital volume & & & & $<0.001$ & & & & 0.002 \\
\hline$<75 \%$ & $5,593(80.7)$ & $3,542(53.6)$ & -0.601 & & $4,946(71.4)$ & $4,510(68.3)$ & -0.067 & \\
\hline$\geq 75 \%$ & $1,339(19.3)$ & $3,061(46.4)$ & & & $1,986(28.6)$ & $2,093(31.7)$ & & \\
\hline
\end{tabular}

Values are presented as number (\%).

CCI, Charlson Comorbidity Index.

ent the overall prevalence of PI surgery in this large cohort sample and to compare the difference of risk of PI surgery according to the type of surgical modality used. The prevalence of PI surgery reported in this study (3.3\%) was lower than those that have been previously reported in the literature $(4 \%-6 \%)$, which might have resulted from the differences in the defini- tion of PI used, the follow-up period, and the inclusion of study population [7,9-11]. Moreover, this study revealed that the rate of PI surgery for patients undergoing the minimally invasive $\mathrm{RP}$ was higher than patients receiving open surgery in the adjusted analysis, which was mainly due to the high PI prevalence of laparoscopic RP. 
Table 2. Crude frequency rate of anti-incontinence procedure by age group

\begin{tabular}{|c|c|c|c|c|c|}
\hline Age group (yr) & No procedure & Any procedure & Sling & Bulking agent & AUS \\
\hline Overall $(\mathrm{n}=13,535)$ & $13,089(96.7)$ & $446(3.3)$ & $247(1.8)$ & $115(0.8)$ & $84(0.6)$ \\
\hline$<55(\mathrm{n}=2,132)$ & $2,082(97.7)$ & $50(2.3)$ & $34(1.6)$ & $9(0.4)$ & $7(0.3)$ \\
\hline $55-64(\mathrm{n}=5,307)$ & $5,165(97.3)$ & $142(2.7)$ & $84(1.6)$ & $35(0.7)$ & $23(0.4)$ \\
\hline $65-74(\mathrm{n}=5,203)$ & $4,984(95.8)$ & $219(4.2)$ & $113(2.2)$ & $59(1.1)$ & $47(0.9)$ \\
\hline$>74(\mathrm{n}=893)$ & $858(96.1)$ & $35(3.9)$ & $16(1.8)$ & $12(1.3)$ & $7(0.8)$ \\
\hline
\end{tabular}

Values are presented as number (\%).

AUS, artificial urinary sphincter.

Table 3. Unadjusted and adjusted absolute risk differences and risk ratios for postprostatectomy incontinence in patients undergoing minimally invasive and open radical prostatectomy, 2008-2010

\begin{tabular}{|c|c|c|c|c|}
\hline & \multicolumn{2}{|c|}{ Radical prostatectomy } & \multirow{2}{*}{$\begin{array}{l}\text { Absolute risk difference } \\
\qquad(\%)(95 \% \mathrm{CI})\end{array}$} & \multirow{2}{*}{$\begin{array}{l}\text { Risk ratio } \\
(95 \% \mathrm{CI})\end{array}$} \\
\hline & $\begin{array}{c}\text { Open } \\
(n=6,932)\end{array}$ & $\begin{array}{l}\text { Minimally invasive } \\
\qquad(\mathrm{n}=6,603)\end{array}$ & & \\
\hline Unadjusted & $211(3.0)$ & $235(3.6)$ & $0.5(-0.8$ to 1.8$)$ & $1.17(0.81-1.69)$ \\
\hline $\begin{array}{l}\text { Adjusted by inverse probability } \\
\text { of treatment weighting }\end{array}$ & $210(3.0)$ & $319(4.8)$ & $1.8(0.3-3.4)^{\star}$ & $1.60(1.16-2.20)^{\star}$ \\
\hline
\end{tabular}

Values are presented as number of events (\%) unless otherwise indicated.

$\mathrm{CI}$, confidence interval.

Unadjusted absolute risk difference and risk ratio were analyzed using standard logistic regression model; adjusted absolute risk difference and risk ration were analyzed using inverse probability of treatment weighting weighted gamma distribution model.

${ }^{*} \mathrm{P}<0.05$, statistically significant differences and open procedure was referenced.

Table 4. Unadjusted and adjusted absolute risk differences and risk ratios for postprostatectomy incontinence in patients undergoing robot-assisted, laparoscopic, perineal, and retropubic radical prostatectomy, 2008-2010

\begin{tabular}{|c|c|c|c|c|c|c|}
\hline & \multicolumn{4}{|c|}{ Radical prostatectomy } & \multirow{2}{*}{$\begin{array}{l}\text { Absolute risk } \\
\text { difference } \\
(\%)(95 \% \mathrm{CI})\end{array}$} & \multirow{2}{*}{$\begin{array}{c}\text { Risk ratio } \\
(95 \% \mathrm{CI})\end{array}$} \\
\hline & $\begin{array}{l}\text { Retropubic } \\
(n=5,173)\end{array}$ & $\begin{array}{c}\text { Perineal } \\
(\mathrm{n}=1,759)\end{array}$ & $\begin{array}{l}\text { Laparoscopic } \\
\quad(n=503)\end{array}$ & $\begin{array}{l}\text { Robot-assisted } \\
\qquad(\mathrm{n}=6,100)\end{array}$ & & \\
\hline \multicolumn{7}{|l|}{ Unadjusted } \\
\hline Perineal vs. retropubic & $189(3.7)$ & $22(1.3)$ & & & $-2.4(-3.4$ to -1.4$)$ & $0.34(0.21-0.56)^{*}$ \\
\hline Laparoscopic vs. retropubic & $189(3.7)$ & & $31(6.2)$ & & $2.5(-0.9$ to 5.9$)$ & $1.69(0.97-2.95)$ \\
\hline Robot-assisted vs. retropubic & $189(3.7)$ & & & $204(3.3)$ & $-0.3(-1.6$ to 0.9$)$ & $0.92(0.63-1.33)$ \\
\hline \multicolumn{7}{|c|}{ Adjusted by inverse probability of treatment weighting } \\
\hline Perineal vs. retropubic & $222(4.3)$ & $10(0.6)$ & & & $-3.8(-4.9$ to -2.6$)$ & $0.13(0.07-0.23)^{*}$ \\
\hline Laparoscopic vs. retropubic & $189(3.7)$ & & $43(8.6)$ & & $4.9(-0.8$ to 10.7$)$ & $2.36(1.20-4.64)^{\star}$ \\
\hline Robot-assisted vs. retropubic & $195(3.8)$ & & & $239(3.9)$ & $0.1(-1.1$ to 1.4$)$ & $1.04(0.76-1.42)$ \\
\hline
\end{tabular}

Values are presented as number of events (\%) unless otherwise indicated.

CI, confidence interval.

Unadjusted absolute risk difference and risk ratio were analyzed using standard logistic regression model; adjusted absolute risk difference and risk ration were analyzed using inverse probability of treatment weighting weighted gamma distribution model.

${ }^{\star} \mathrm{P}<0.05$, statistically significant differences and open procedure was referenced.

Out study has several strengths, including the utilization of a population-based dataset and comparison of RP surgical ap- proach. Considering the time-dependent clinical manifestation of PI after RP, it is reasonable to use the objective definition of 
the need for an anti-incontinence procedure as a representation of the most bothersome or severe urinary incontinence. Two previous large observational studies have shown similar prevalence rates of PI necessitating intervention using the similar definition as that used by our study. Kim et al. [12] reported that the prevalence of PI was 6\%, according to Surveillance, Epidemiology, and End Results (SEER) cancer registry data linked with Medicare claims from 2000 to 2007. Similarly, Lowrance et al. [13] reported the prevalence of PI to be 5.45\% using SEER cancer registry data linked with Medicare claims from 2003 to 2005.

Older age is strongly associated with the prevalence of PI, and previous large studies using Medicare data have been limited to patients 65 years older, whereas our study is more inclusive of younger ages $[12,13]$. Further, these studies of older patients have demonstrated slightly increasing PI rates with prolonged follow-up, which is likely attributable to other factors that influence incontinence (overactive bladder, sarcopenia) as men get older $[11,14]$. Therefore, our study provides critical information for patients under 65 years of age requiring RP surgery since it includes a substantially longer follow-up period than previous studies. Only 2 studies have shown a follow-up duration similar to our study, with a median of 20 months and a minimal duration of 24 months [12,15].

To date, there has been controversy over whether minimally invasive procedures including robotic-assisted or laparoscopic $\mathrm{RP}$ are equivalent in terms of risk of PI compared to open RP. Due to the lack of randomized, controlled investigations comparing RP surgical procedures, it is difficult to conclude which surgical type (s) is superior. Several studies confirm that open $\mathrm{RP}$ and robotic-assisted RP have equivalent outcomes oncological outcomes [16]. Recently, Sujenthiran et al. [15], using a large administrative database, reported that robotic-assisted RP showed significantly better functional outcomes postoperatively, including nonspecific urinary and stricture-related complications, compared to open and laparoscopic RP. However, the definition of urinary complications used in this study included intervention codes that were not specific to PI, but included all types of urologic procedures of the urethra, prostate, and bladder. Other studies have shown that robotic-assisted RP shows lower rates of perioperative complications (blood transfusions, shorter time of catheter use postoperatively, shorter length of hospital stay), postoperative complications (PI and ED), and positive surgical margin rates compared to open approaches $[17,18]$. O'Neil et al. [19] reported that, relative to open RP, pa- tients undergoing robotic-assisted RP had higher rates of early recovery of sexual function and continence. However, Barry et al. [17] reported that robotic-assisted RP was not associated with better outcomes compared to PI in their retrospective cohort analyses. Moreover, Hu et al. [20] reported that the use of minimally invasive procedures including either robotic-assisted or laparoscopic RP increased 5-fold from 9.2\% at 2003 to $43.2 \%$ at 2006-2007, with patients receiving minimally invasive procedures reporting increased genitourinary complications (4.7\% vs. $2.1 \%$ ) and a greater likelihood of a PI diagnosis (15.9 vs. 12.2 per 100 person-years, $\mathrm{P}=0.02$ ).

When comparing the prevalence of PI procedures between laparoscopic and open RP, our data also support previous reports suggesting that laparoscopic RP yields the highest prevalence of necessitating PI procedures. Huang et al. [21] reported lower rates of PI and ED for robotic-assisted RP compared with laparoscopic RP in a recent meta-analysis. Lowrance et al. [13] reported that PI necessitating anti-incontinence intervention in $5.0 \%$ of open RP cases and $6.2 \%$ of laparoscopic RPs, respectively.

One unique feature of our study population is that it includes a substantial number of men who underwent perineal RP. Perineal RP yielded the lowest prevalence of PI surgery significantly lower relative to the other $3 \mathrm{RP}$ modalities. Our results support the studies by Bishoff et al. [22] and Harris [23] which both reported that perineal RP is associated with the lowest rates of PI and was more cost-effective compared with retropubic RP. The lower rates of PI surgery we observed in perineal RP might explain the recent finding that use of a posterior approach is associated with better continence and potency rates compared to conventional anterior approaches [24].

Our study does have several limitations. First, as this data originates from one geographical area (Florida), the results might be influenced by local practice patterns that limit generalization to other populations. Second, limited information exists regarding clinically-relevant variables including disease characteristics, surgeon volume, and factors influencing patient choice of therapy, surgical approach, and surgeon that could influence their outcomes with regard to PI, as well as their later choice to have a procedure for PI. Third, although we demonstrated risk difference and $\mathrm{RR}$, additional analyses investigating the possible causative factors of PI requiring surgery were not possible. Possible reasons for higher rates of PI surgery in the minimally invasive RP group include a greater expectation regarding clinical outcomes for robotic-assisted RP relative to 
open RP, a steeper learning curve for robotic-assisted RP, and the emergence of new robotic-assisted RP surgical techniques [25]. Fourth, conversion rates from minimally invasive to open $\mathrm{RP}$ were not captured, although previously reported rates are low [26]. Fifth, the actual prevalence rate of severe and bothersome PI is under captured through procedural codes because of coding errors and since some men might not elect for surgery despite having severe symptoms. Sixth, this study could not consider surgical factors including postoperative membranous urethral length. Recently, Cho et al. [27] highlighted the importance of saving the membranous urethral length as long as possible in terms of continence recovery. Finally, long-term data are necessary in order to compare longer functional outcomes, especially those related to QoL, between open and minimally invasive procedures

In conclusion, among patients undergoing RP for PCa, the prevalence of PI surgery is not negligible. Patients undergoing minimally invasive RP had higher adjusted rates for PI surgery compared to open approaches, which was attributed to high rate of PI surgery following laparoscopic approach and low rate of PI surgery following perineal approach. More studies are needed to establish strategies to reduce the rate of PI surgery after RP.

\section{AUTHOR CONTRIBUTION STATEMENT}

- Conceptualization: IGJ, THB, JB, BC

- Data curation: JHK, YK, THB

- Formal analysis: $J H K, Y K, B C$

- Funding acquisition: $J H K$

- Project administration: IGJ, BC

- Writing-original draft: JHK, IGJ

- Writing-review \& editing: $J H K, Y K, T H B, J B, B C$

\section{ORCID}

$\begin{array}{ll}\text { Jae Heon Kim } & 0000-0002-4490-3610 \\ \text { In Gab Jeong } & 0000-0003-4093-832 \mathrm{X} \\ \text { Yash S. Khandwala } & 0000-0001-9336-7407 \\ \text { Tina Hernandez-Boussard } & 0000-0001-6553-3455 \\ \text { James D. Brooks } & 0000-0003-4521-7505 \\ \text { Benjamin I. Chung } & 0000-0001-9660-2732\end{array}$

\section{REFERENCES}

1. Yamamoto S, Kawakami S, Yonese J, Fujii Y, Urakami S, Kitsukawa $\mathrm{S}$, et al. Long-term oncological outcome in men with T3 prostate cancer: radical prostatectomy versus external-beam radiation therapy at a single institution. Int J Clin Oncol 2014;19:1085-91.

2. Freedland SJ, Humphreys EB, Mangold LA, Eisenberger M, Dorey FJ, Walsh PC, et al. Risk of prostate cancer-specific mortality following biochemical recurrence after radical prostatectomy. JAMA 2005;294:433-9.

3. Loughlin KR, Prasad MM. Post-prostatectomy urinary incontinence: a confluence of 3 factors. J Urol 2010;183:871-7.

4. Penson DF, Feng Z, Kuniyuki A, McClerran D, Albertsen PC, Deapen $D$, et al. General quality of life 2 years following treatment for prostate cancer: what influences outcomes? Results from the prostate cancer outcomes study. J Clin Oncol 2003;21:1147-54.

5. Majoros A, Bach D, Keszthelyi A, Hamvas A, Romics I. Urinary incontinence and voiding dysfunction after radical retropubic prostatectomy (prospective urodynamic study). Neurourol Urodyn 2006;25:2-7.

6. Trofimenko V, Myers JB, Brant WO. Post-prostatectomy incontinence: how common and bothersome is it really? Sex Med Rev 2017;5:536-43.

7. Ficarra V, Novara G, Rosen RC, Artibani W, Carroll PR, Costello A, et al. Systematic review and meta-analysis of studies reporting urinary continence recovery after robot-assisted radical prostatectomy. Eur Urol 2012;62:405-17.

8. Tienza A, Robles JE, Hevia M, Algarra R, Diez-Caballero F, Pascual JI. Prevalence analysis of urinary incontinence after radical prostatectomy and influential preoperative factors in a single institution. Aging Male 2018;21:24-30.

9. Bauer RM, Gozzi C, Hubner W, Nitti VW, Novara G, Peterson A, et al. Contemporary management of postprostatectomy incontinence. Eur Urol 2011;59:985-96.

10. Holm HV, Fossa SD, Hedlund H, Schultz A, Dahl AA. How should continence and incontinence after radical prostatectomy be evaluated? A prospective study of patient ratings and changes with time. J Urol 2014;192:1155-61.

11. Stanford JL, Feng Z, Hamilton AS, Gilliland FD, Stephenson RA, Eley JW, et al. Urinary and sexual function after radical prostatectomy for clinically localized prostate cancer: the Prostate Cancer Outcomes Study. JAMA 2000;283:354-60.

12. Kim PH, Pinheiro LC, Atoria CL, Eastham JA, Sandhu JS, Elkin EB. Trends in the use of incontinence procedures after radical prostatectomy: a population based analysis. J Urol 2013;189:602-8. 
13. Lowrance WT, Elkin EB, Jacks LM, Yee DS, Jang TL, Laudone VP, et al. Comparative effectiveness of prostate cancer surgical treatments: a population based analysis of postoperative outcomes. J Urol 2010;183:1366-72.

14. Sacco E, Prayer-Galetti T, Pinto F, Fracalanza S, Betto G, Pagano F, et al. Urinary incontinence after radical prostatectomy: incidence by definition, risk factors and temporal trend in a large series with a long-term follow-up. BJU Int 2006;97:1234-41.

15. Sujenthiran A, Nossiter J, Parry M, Charman SC, Aggarwal A, Payne H, et al. National cohort study comparing severe mediumterm urinary complications after robot-assisted vs laparoscopic vs retropubic open radical prostatectomy. BJU Int 2018;121:445-52.

16. El-Hakim A, Leung RA, Tewari A. Robotic prostatectomy: a pooled analysis of published literature. Expert Rev Anticancer Ther 2006;6:11-20.

17. Barry MJ, Gallagher PM, Skinner JS, Fowler FJ Jr. Adverse effects of robotic-assisted laparoscopic versus open retropubic radical prostatectomy among a nationwide random sample of medicareage men. J Clin Oncol 2012;30:513-8.

18. Haglind E, Carlsson S, Stranne J, Wallerstedt A, Wilderang U, Thorsteinsdottir T, et al. Urinary incontinence and erectile dysfunction after robotic versus open radical prostatectomy: a prospective, controlled, nonrandomised trial. Eur Urol 2015;68:21625.

19. O’Neil B, Koyama T, Alvarez J, Conwill RM, Albertsen PC, Cooperberg MR, et al. The comparative harms of open and robotic prostatectomy in population based samples. J Urol 2016;195:321-9.

20. Hu JC, Gu X, Lipsitz SR, Barry MJ, D’Amico AV, Weinberg AC, et al. Comparative effectiveness of minimally invasive vs open radical prostatectomy. JAMA 2009;302:1557-64.

21. Huang X, Wang L, Zheng X, Wang X. Comparison of perioperative, functional, and oncologic outcomes between standard laparoscopic and robotic-assisted radical prostatectomy: a systemic review and meta-analysis. Surg Endosc 2017;31:1045-60.

22. Bishoff JT, Motley G, Optenberg SA, Stein CR, Moon KA, Browning $\mathrm{SM}$, et al. Incidence of fecal and urinary incontinence following radical perineal and retropubic prostatectomy in a national population. J Urol 1998;160:454-8.

23. Harris MJ. The anatomic radical perineal prostatectomy: an outcomes-based evolution. Eur Urol 2007;52:81-8.

24. Menon M, Dalela D, Jamil M, Diaz M, Tallman C, Abdollah F, et al. Functional recovery, oncologic outcomes and postoperative complications after robot-assisted radical prostatectomy: an evidencebased analysis comparing the Retzius sparing and standard approaches. J Urol 2018;199:1210-7.

25. Schroeck FR, Krupski TL, Sun L, Albala DM, Price MM, Polascik TJ, et al. Satisfaction and regret after open retropubic or robot-assisted laparoscopic radical prostatectomy. Eur Urol 2008;54:785-93.

26. Sharma V, Meeks JJ. Open conversion during minimally invasive radical prostatectomy: impact on perioperative complications and predictors from national data. J Urol 2014;192:1657-62.

27. Cho DS, Choo SH, Kim SJ, Shim KH, Park SG, Kim SI. Postoperative membranous urethral length is the single most important surgical factor predicting recovery of postoperative urinary continence. Urol Oncol 2020;38:930.e7-930.e12. 